Dos s i e r

$>$ La fiscalité locale

regards comparatifs

Colloque de Lyon

10 octobre 2019

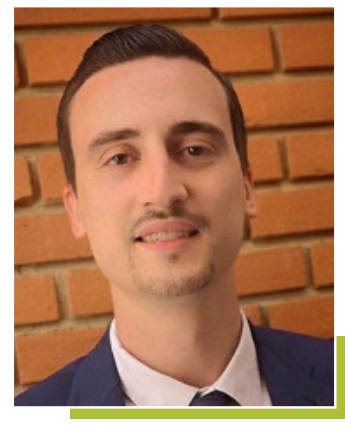

Léo GARCIA

Doctorant en droit public,Institut Maurice Hauriou,

Université de Toulouse 1 capitole

\title{
L'intervention du droit souple financier : quelles conséquences pour la liberté et la solidarité intercommunale?
}

\begin{abstract}
Mots-clés
finances locales - intercommunalité - EPCI - pacte financier et fiscal subvention - droit souple
\end{abstract}

Les instruments de droit souple sont de plus en plus présents dans l'arsenal juridique des établissements publics de coopération intercommunale à fiscalité propre (EPCI-FP). L'utilisation croissante de ces outils est issue d'une volonté des exécutifs intercommunaux de bénéficier d'une plus grande liberté d'adaptation de leur politique financière au territoire. Mais leur utilisation peut remettre en cause des aspects de la solidarité territoriale sur le périmètre de l'EPCI-FP.

extension du droit souple dans les collectivités territoriales répond à un besoin d'assouplissement du cadre normatif. Toutefois, les dispositifs qui élargissent les marges de manœuvre financière des $\mathrm{EPCl}$ ne sont pas sans limites.

Le Doyen Carbonnier estimait que le « droit est plus grand que la règle de droit »' . Une conception des sources juridiques large qui atteste la présence de deux catégories de droit : un droit " dur » considéré comme normatif, contraignant, obligatoire ${ }^{2}$ et pourvu de sanctions, cohabite avec un droit "souple ", adaptable et flexible ${ }^{3}$, prescrivant des bonnes pratiques et malléable ${ }^{4}$.

Pactes, chartes, lignes directrices, grilles de lecture, recommandations, avis, sont tout autant de dispositifs de droit souple qui font peser une contrainte morale sur leurs auteurs et leurs
Jean Carbonnier, « Droit et non droit » in Flexible droit, LGDJ, $10^{\circledR}$ édition, 2001, p.22

${ }^{2}$ Mireille Delmas-Marty, Le

flou du droit, Paris, Presses Universitaires de France, coll. Quadrige, 2004, 338 p.

${ }^{3}$ Christian Vigouroux, "Conclusion », in Séminaire Aca-Europe : Droit souple normes juridiques et sources du droit, Conseil d'État, 18 décembre 2013

${ }^{4}$ Nathalie Laval-Mader, « Vers la juridictionnalisation du droit souple des collectivités territoriales ?», BJCL, octobre 2018, n 10/18, pp. 696-701. 
o s s i e r

$>$ La fiscalité locale : regards comparatifs Colloque de Lyon 10 octobre 2019
5 Jean-Marc Pastor, "Acte III, scène finale? ", JCPA, 20 octobre $2014, n^{\circ} 42$, pp. $17-21$

De la sécurité juridique, Étude annuelle 1991, n 43 , Coll, Les rapports du Conseil d'État, La Documentation Française 1991

7 Ibid, p. 256

Sécurité juridique et complexité du droit, Étude annuelle 2006, n 57, Coll, Les rapports du Conseil d'État,

La Documentation Française 2006, $411 p$.

Décision n²004-500 DC du 29 juillet 2004 : Damien Chamussy, « Le Conseil constitutionnel et la qualite de la législation", Revue du droit public et de la science politique en France et à l'étranger, novembredécembre 2004, $n^{\circ} 6$ p. $1739-1760$ destinataires sans pour autant créer de droits ou d'obligations. Ces outils se développent de manière exponentielle à côté du droit dur dans toutes les branches du droit, jusque dans celui qui régit les finances intercommunales, et notamment celles des EPCl-FP. Ces dispositifs vont leur donner la possibilité de mettre en place une politique financière propre à leurs besoins, adaptée et moins figée par les textes normatifs. Ces EPCI-FP vont pouvoir bénéficier d'une liberté accrue pour déterminer leurs politiques financières sur leur territoire.

Mais l'utilisation de ces différents instruments de droit souple peut s'avérer contreproductive. Leur faible force contraignante et l'excès de liberté qui peut en découler peut remettre en cause le projet de territoire d'un EPCI-FP ou rendre plus difficile l'accès à des ressources financières pour les acteurs du territoire intercommunal.

Le développement du droit souple dans les finances des EPCl-FP donne donc matière à réflexions sur ses conséquences en matière de libertés locales et de solidarité territoriale intercommunale. II sera traité, dans un premier temps, le développement progressif du droit souple dans les finances intercommunales (I.) et, dans un deuxième temps, il sera démontré que ce droit souple est une garantie, mais une garantie à nuancer, d'une certaine liberté et solidarité territoriale sur les territoires intercommunaux (II.)

\section{Le développement progressif du droit souple dans les finances intercommunales}

La difficile conciliation entre, d'un côté, la volonté de développer les adaptations locales et lutter contre la rigidité d'un droit dur pouvant être parfois un handicap dans le bon fonctionnement des collectivités territoriales, et, d'un autre côté, de garantir la sécurité juridique et la légitimité du droit dur dans l'arsenal juridique va être un frein, dans un premier temps, à I'utilisation du droit souple par les échelons locaux et les EPCl-FP (A). Mais les rationalisations et les fusions récentes de ces EPCl-FP qui ont été opérées notamment sous l'empire de ce qui semble être le troisième acte de la décentralisation ${ }^{5}$ ont fait naitre un besoin $d^{\prime}$ 'assouplissement normatif (B).

\section{A. La difficile acceptation du droit souple : un frein à son utilisation par les collectivités territoriales}

Il y a une opposition, qui peut être qualifiée de classique, entre ceux qui ne jurent que par le droit dur et ceux qui croient en la possibilité et en la nécessité d'utiliser du droit souple. C'est, en partie, cet affrontement doctrinal sur la place du droit souple dans notre arsenal juridique qui a freiné son développement et son utilisation par les collectivités territoriales et les EPCI-FP.

Les critiques du droit souple, exprimées par une large majorité de la doctrine ou des juridictions, se sont cristallisées principalement autour de deux inquiétudes avant d'être progressivement accepté : la crainte de voir l'insécurité juridique se développer et l'application d'un droit qui serait illégitime.

\section{La crainte d'une croissance de l'insécurité juridique}

La grande majorité de la doctrine et des juridictions regrettaient la dégradation de la qualité du droit avec l'intervention d'instruments et d'actes de droit souple. Cela compromettrait la qualité du droit dur par une atteinte à sa clarté, à sa lisibilité et à la portée des actes pour leurs destinataires, ne sachant pas si cet acte créé véritablement des droits et des obligations.

Le Conseil d'État a, par deux fois, relevé ces vicissitudes. Tout d'abord en 1991 dans son rapport intitulé «De la sécurité juridique " ${ }^{6}$ qui dénonçait le développement, dans les lois et les décrets, d'énoncés peu ou pas normatifs et qui qualifiait le droit souple comme des «textes d'affichage, un droit mou, un droit flou, un droit à l'état gazeux $»^{7}$. Ensuite en 2006, dans son rapport public annuel intitulé "Sécurité juridique et complexité du droit $»^{8}$, où il soulignait la permanence des pratiques qu'il décriait 15 ans plus tôt.

Le Conseil Constitutionnel a également pu exprimer cette inquiétude, notamment en censurant certaines dispositions législatives sans portées normatives. Ce fût le cas dans sa décision du 29 juillet 2004 sur la loi organique relative à I'autonomie financière des collectivités territoriales ${ }^{9}$ où le Conseil Constitutionnel énonce dans le considérant de principe que « la loi a pour vocation d'énoncer des règles et doit par suite être revêtue d'une portée normative ».

Après les critiques relatives à l'insécurité du droit souple, une partie de la doctrine et des juridic- 
tions vont aussi dénoncer l'application d'un droit qui serait, à leurs yeux, illégitime.

\section{L'application d'un droit qui serait illégi-} time

Pour ses opposants, la mise en place d'instruments de droit souple et des actes qui en découlent contournerait les institutions chargées d'élaborer les règles de droit dur, à savoir les institutions parlementaires. Parmi elles, le Parlement Européen a réagi en adoptant en 2007 une résolution ${ }^{10}$ pour dénoncer ce contournement du droit dur par les instruments juridiques non-contraignants.

Le procès en illégitimité du droit souple va donc s'exprimer en pointant du doigt l'incompétence des acteurs publics qui le créé et le manipule. Car pour ceux qui utilisent le droit dur, le droit souple serait une possibilité donnée à des acteurs publics pour passer outre ces règles de compétences législatives.

Certains de ces acteurs publics, notamment les collectivités territoriales qui ne cessent de plaider pour un renforcement de la décentralisation et des libertés locales, vont venir concurrencer la compétence législatrice du Parlement en créant des instruments ad-hoc et utiliser des actes de droit souple face à un État qualifié de plus en plus comme Jacobin.

\section{La progressive acceptation du droit} souple

Outre le symbolique changement d'appellation, passant d'une péjorative dénomination de droit mou à une dénomination moins négative de droit souple, une partie de la doctrine et des juridictions ont commencé à accepter la présence de ce droit malléable au côté d'un droit plus rigide et ont surtout saisi l'intérêt d'y avoir recours.

C'est le cas du Conseil d'État qui a changé sa vision sur cette question. Après avoir décrié le développement des instruments de droit souple, notre plus haute juridiction administrative s'est ravisée. Le Conseil d'État publiera un rapport en $2013^{11}$ où il prend acte de l'omniprésence du droit souple et prend une position favorable, mais mesurée, sur l'utilité du droit souple avec les conditions de son efficacité tout en présentant les éventuels risques qu'il présente. Dans ce rapport, le Conseil d'État présente également des propositions d'utilisation et une "doctrine" d'emploi du droit souple.

Les collectivités territoriales vont profiter de ce mouvement d'acceptation du droit souple au côté du droit dur pour développer et mettre en place des instruments de droit souple dans leur arsenal juridique, et ce de manière plus assumée.
Ce fût notamment le cas pour les EPCI-FP qui ont ressenti un besoin nécessaire d'assouplissement normatif, notamment au moment de l'Acte III de la décentralisation.

\section{B. Un besoin nécessaire d"assouplis- sement normatif né de l'Acte III de la décentralisation}

Cette nouvelle étape dans la réforme territoriale, qualifiée aussi de troisième Acte de la décentralisation, est identifiable par plusieurs lois venues réformer l'organisation territoriale de notre pays. Deux textes majeurs sont venus bouleverser l'organisation et le fonctionnement de l'intercommunalité et plus particulièrement des EPCl-FP, ce qui n'a pas été sans incidence sur le plan financier. II s'agit de la Loi MAPTAM du 27 janvier $2014^{12}$ et de la Loi NOTRe du 7 août $2015^{13}$.

Outre l'annulation de la suppression de la clause de compétence générale pour les communes et les départements ou la création des collectivités chefs de file dans l'exercice d'une compétence, la loi MAPTAM avait comme objectif d'affirmer la place donnée aux métropoles dans le paysage institutionnel local. Une affirmation qui s'est traduite, d'une part par la création de nouvelles métropoles dont la Métropole du Grand Paris, la Métropole de Lyon, la métropole d'Aix-Marseille-Provence et de 10 autres par décret et, d'autre part par l'exercice, pour ces récentes métropoles, de compétences stratégiques.

La loi NOTRe a quant à elle modifié la carte intercommunale, notamment en relevant le seuil du nombre d'habitants pour les communautés de communes (15000 habitants au lieu de 5000 , sauf dérogations notamment pour les territoires de montagne ou à faible densité). Pour respecter ce seuil, des Schémas Départementaux de Coopération Intercommunale ont été établis sous la houlette du préfet et en association avec les élus locaux pour prescrire des fusions entre plusieurs EPCI-FP. Leur nombre a été abaissé de 2133 en 2015 à 1258 en 2019. A noter que le nombre de communautés de communes a diminué de $47 \%$ en 6 ans.

Ces deux lois ont donc fait naitre de grandes métropoles dans le paysage intercommunal et a permis la création d'EPCl-FP qui ont pu être qualifiées par une partie de la doctrine d'EPCl «XL » ${ }^{14}$. XXL sur le plan géographique tout d'abord, car la superficie moyenne des communautés de communes a logiquement augmenté en raison des fusions entre plusieurs EPCl-FP. $X X L$ ensuite, sur le plan de la composition des conseils communautaires. L'intégration de nouvelles communes membres a engendré une aug-

\footnotetext{
${ }^{10}$ Résolution du Parlement européen du 4 septembre 2007 sur les implications juridiques et institutionnelles du recours aux instruments juridiques non contraignants (soft law) (2007/2028(INI))

${ }^{11}$ Le droit souple, Étude annuelle 2013, n 64, Coll, Les rapports du Conseil d'État, La Documentation Française, 2013, $297 p$.

${ }^{12}$ Loi n ${ }^{\circ} 2014-58$ du 27 janvier 2014 de modernisation de I'action publique territoriale et d'affirmation des métropoles.

${ }^{13}$ Loi n 2015-991 du 7 août 2015 portant nouvelle organisation territoriale de la République.

${ }^{14}$ Florence Crouzatier-Durand, "Réflexions sur la légitimité d'action au sein des intercommunalités XXL ", BJCL, 1er juillet 2017, $n^{\circ} 7-8$, p.508-511; Jean-Marc Pastor, "Nouveau calendrier pour les intercommunalités XXL ?" AJDA, 18 avril 2016, n 13 p. 694
} 
o s s i e r

$>$ La fiscalité locale : regards comparatifs Colloque de Lyon 10 octobre 2019 mentation du nombre de sièges d'élus communautaires afin que chacune de ces nouvelles communes membres soient représentées. XXL également sur le plan des compétences exercées, car la loi NOTRe a dévolu de nouvelles compétences à ces EPCl-FP. Cette loi leur en a aussi donné la possibilité d'en exercer à titre optionnel ou facultatif. Enfin, XXL sur le plan financier, puisque les budgets de ces nouveaux EPCI-FP fusionnés seront beaucoup plus importants, que cela soit en recettes comme en dépenses, en fonctionnement comme en investissement.

C'est pour répondre aux récentes problématiques qui ont pu naitre de cette nouvelle carte intercommunale que les élus et les agents ont vu I'intérêt de gagner en souplesse dans la mise en œuvre du droit auquel les EPCI-FP sont soumis et dans l'utilisation de leurs ressources financières et de leurs leviers fiscaux. Ces problématiques, relayées par les élus locaux et le législateur, ont trait notamment à l'éloignement de certaines communes du centre de décision, au poids politique et administratif écrasant de la commune-centre, à la difficulté à trouver un consensus entre les territoires urbains et les territoires ruraux ou encore au sentiment des communes membres se sentant dépossédées de leurs moyens d'action et de leurs compétences stratégiques au profit des EPCl-FP.

Il y a donc une volonté politique et administrative de la part des EPC-FP d'être moins corsetés par certains textes législatifs et règlementaires pouvant être considérés comme rigides. D'autre part, ces acteurs intercommunaux souhaitent mettre en avant la confiance donnée à l'intelligence territoriale ${ }^{15}$ à travers les élus communautaires et les services intercommunaux. Cette volonté vise à favoriser l'adaptation des politiques financières aux besoins et aux équilibres politiques de chaque EPCl-FP.

Face à ce double constat - acceptation du droit souple dans l'arsenal juridico-financier des EP$\mathrm{Cl-FP}$ et nécessité d'utiliser ce droit souple par les acteurs locaux intercommunaux - il est pertinent d'expliquer en quoi ce droit souple peut garantir une relative liberté et solidarité financière sur les territoires intercommunaux.

\section{La garantie nuancée d'une certaine liberté et solidarité financière sur les territoires intercommunaux}

Le développement du droit souple dans l'arsenal juridique des collectivités territoriales et des EP$\mathrm{Cl}-\mathrm{FP}$ va permettre assurément d'accroitre leurs marges de manœuvres financières (A). Mais la diffusion de ce droit souple par l'utilisation des différents dispositifs financiers qui sont à la disposition des EPCI-FP peut entrainer des effets potentiellement contreproductifs au regard des objectifs et des buts initiaux conférés à ces dispositifs (B).

\section{A. L'accroissement des marges de manœuvres financières pour les EP- CImFP}

L'assouplissement de certaines dispositions législatives de droit commun en matière financière par le droit souple va intervenir dans les finances intercommunales pour remplir principalement l'objectif d'adaptation et de modulation des dispositifs financiers au regard des besoins, des spécificités et des caractéristiques financières du territoire intercommunal et des communes membres.

Cet accroissement des marges de manœuvre financières pour les EPCI-FP par le droit souple in- tervient grâce à plusieurs dispositifs. Cette étude se portera sur deux d'entre eux : le pacte financier et fiscal et les outils méthodologiques dans les attributions de subventions.

Les pactes financiers et fiscaux intercommunaux pour concrétiser financièrement les projets de territoire

Avec la baisse des dotations de l'État, la stagnation de la capacité contributive des ménages, l'absence de dynamique des assiettes fiscales et la modification de la carte intercommunale, les EPCl-FP et leurs communes membres ont dû s'interroger sur une nouvelle répartition des charges et des ressources financières sur le territoire intercommunal.

Ces nouvelles perspectives financières vont motiver les EPCl-FP à signer avec leurs communes membres des pactes financiers et fiscaux. Le législateur ne donne pas de définition précise de ces documents, excepté l'objectif de réduire les disparités de charges et de recettes entre les communes membres des EPCl-FP'16. Mais les associations d'élus et plus particulièrement l'Assemblée des Communautés de France (AdCF) va proposer des modèles de pactes financier et fis- 
caux et expliciter leurs contenus et leurs objectifs. Les pactes financiers sont considérés par l'AdCF comme des alliés pour les nouveaux mandats locaux ${ }^{17}$ et une pièce maitresse dans l'accompagnement, la mise en place et le financement du projet de territoire porté par l'intercommunalité.

Les pactes financiers et fiscaux sont obligatoires pour les communautés urbaines, les métropoles, la métropole de Lyon ou lorsque l'EPCI-FP est signataire d'un contrat de ville ${ }^{18}$ mais restent, par déduction, facultatifs pour les communautés de communes et les communautés d'agglomération qui n'en sont pas signataires. La souplesse de ce dispositif réside dans son aspect optionnel et dans la liberté des EPCI-FP d'établir son contenu pour atteindre l'objectif fixé par le Code général des impôts.

Ce pacte financier et fiscal est un document qui va permettre de garantir l'équilibre financier et fiscal de l'ensemble intercommunal au regard du projet de territoire qui sera porté par l'exécutif de l'EPCI-FP. C'est ce document de planification financière et de gestion budgétaire qui va donner le cap financier à suivre par l'intercommunalité. Les directions à suivre et proposées par le pacte financier et fiscal sont, d'une part, à la destination des élus et de l'exécutif intercommunal dans la proposition et le vote de délibérations, et d'autre part à la destination des services et des agents pour mettre en application et concrétiser financièrement ces délibérations.

Ce pacte financier et fiscal va donc donner aux EPCl-FP un cadre dans lequel ils pourront user de toute la latitude pour organiser leur fonctionnement financier. C'est un document qui va donner, dans le respect des règles de droit dur, davantage de liberté à ces structures intercommunales par la mise en œuvre de dispositifs financiers adaptés et calibrés à la situation financière du territoire de I'EPCI-FP.

\section{Les instruments pour guider les EPCI-FP dans I'attribution de subventions}

Les EPCl-FP peuvent également adopter un instrument de droit souple pour exercer leur compétence d'attribution de subventions afin d'aider des associations, des clubs sportifs et d'autres collectivités dans leur fonctionnement ou pour soutenir financement des projets réalisés sur le territoire.

Charte de versement, grille indicative, convention ou protocole, ces instruments de cadrage dans l'attribution de subventions ont des dénominations très diverses. Mais l'objectif est très régulièrement le même : fixer les règles de modalité d'attribution des subventions.

Ces documents vont être de véritables modes d'emplois, des guides dans la manière dont les subventions seront attribuées par l'EPCl-FP. Pour guider les acteurs intercommunaux dans le versement de subventions, ces documents seront la plupart du temps accompagnés de critères d'attribution.

Ces critères peuvent être très variés et vont témoigner de la grande liberté dont dispose les EPCl-FP dans leur politique d'attribution de subventions. Ces critères peuvent être liés tout d'abord au nombre d'adhérents. Par exemple, plus une association aura d'adhérents, plus elle pourra prétendre à percevoir une subvention importante. Ces critères peuvent également être liés aux résultats obtenus par ces acteurs associatifs. Par exemple, un club de football local qui accède à une division supérieure pourra prétendre à une subvention majorée pour récompenser le résultat sportif. Enfin ces critères peuvent être liés à la politique que souhaite mener l'EPCI-FP. Si ce dernier, dans ses projets prioritaires, souhaite mettre l'accent sur le développement du numérique et des nouvelles technologies, I'attribution d'une subvention à une collectivité territoriale qui réalise un investissement dans ce domaine pourra voir sa subvention bonifiée.

Cette liste de critères, qui est loin d'être exhaustive, prouve la grande diversité de possibilités pour un EPCI-FP d'orienter sa politique d'attribution de subvention.

Ces documents, bien que déterminant dans I'attribution de ressources financières, n'ont aucune valeur juridique, ne sont pas prévus par la loi et sont entièrement facultatifs. Ils sont mis en place dans chaque intercommunalité au regard des spécificités financières du territoire et de la politique que le président de l'EPCI-FP et des élus communautaires souhaitent mener. Ces documents sont surtout utilisés pour rationaliser l'attribution de ces subventions, limiter leur saupoudrage et adapter les sommes à attribuer. Cette attribution se fera au cas par cas au regard du respect des critères qui auront été décidés par I'EPCl-FP.

Mais cette liberté financière laissée aux EPCI-FP par I'utilisation et l'adaptation de ces dispositifs financiers de droit souple doit être nuancée car elle peut faire naître des effets contreproductifs, en contradiction avec les principes de solidarité territoriale. o s s i e r

$>$ La fiscalité locale regards comparatifs Colloque de Lyon 10 octobre 2019
Assemblée Des

Communautés de France et Groupe Caisse des dépôts, Pacte fiscal et financier de solidarité au service du projet de territoire, décembre 2014 , p. 5

${ }^{18} \mathrm{lbid}$. Code général des impôts 
O s s i e r

$>$ La fiscalité locale : regards comparatifs Colloque de Lyon 10 octobre 2019

\section{B. Les limites du droit souple : des ef- fets potentiellement contreproductifs}

Il existe plusieurs cas où le droit souple peut avoir des effets contreproductifs ou présenter des vicissitudes.

C'est par exemple le cas des pactes financiers et fiscaux. Ces pactes de droit souple n'ont rien de contraignants et il est tout à fait concevable et plausible que certaines communes membres de I'EPCI-FP y dérogent en prenant des décisions venant à l'encontre des objectifs et des comportements qui y ont été inscrits.

Il peut arriver par exemple qu'une partie des communes membres d'un EPCl-FP augmentent leurs taux d'imposition des taxes ménages alors que le pacte financier et fiscal auquel ces communes membres sont soumis de par leur appartenance à l'EPCI-FP prévoit un statu quo de ces taux.

Cette absence de contrainte juridique pour les communes à respecter ce pacte financier et fiscal peut donc compromettre son équilibre, sa légitimité et ouvrir la boite de Pandore de l'augmentation de ces taux de taxes ménage pour des communes membres. En effet, aucune sanction juridique n'est prévue en cas de non-respect des engagements inscrits dans le pacte financier et fiscal. La seule sanction que pourraient éventuellement rencontrer les communes membres est une sanction politique. C'est un risque très rare en réalité car cette contrainte politique, voire morale, qui pèse sur les communes membres les poussent à respecter les engagements inscrits dans ce pacte financier et fiscal. Mais si le risque est minime, il n'est pas impossible.
Concernant les documents qui prévoient la manière dont les subventions sont attribuées par I'EPCl-FP, certains présidents d'exécutifs intercommunaux peuvent mettre en place des critères qui pourraient être considérés comme trop restrictifs.

En imposant des critères restreints ou des objectifs difficilement atteignables, certaines associations pourraient, au mieux, avoir des difficultés pour prouver qu'elles entrent dans le cadre des critères, au pire pâtir d'une baisse ou d'une suppression de leur subvention communautaire. La liberté locale que confèrent ces documents dans I'attribution des subventions peut donc avoir des conséquences financières préjudiciables pour les acteurs associatifs et donc porter atteinte à la solidarité, à la cohésion et à la vie du territoire.

Un autre risque existe pour certaines associations. Il est parfaitement imaginable qu'un président d'EPCI-FP s'abrite derrière des critères restrictifs dans l'attribution d'une subvention pour priver de ces subventions certaines associations qui pourraient être critiques envers lui, sa gestion et ses projets.

Au regard de la considération que pouvait avoir généralement la doctrine ${ }^{19}$ du droit souple, son acceptation dans les finances des EPCl-FP est une révolution car il provoquait des levées de boucliers quasi-unanimes. De plus en plus toléré et désormais accepté, son utilisation semble être aujourd'hui le fruit d'une évolution relativement logique au regard de la volonté, pour les EPCI-FP et les collectivités territoriales, de disposer de davantage de liberté et de possibilité d'adaptation dans la gestion de leurs finances.
19 V. Mustapha Mekki in Association Henri Capitant des amis de la culture juridique française, Le droit souple, Paris, Dalloz, coll. Thèmes et commentaires, 2009, 179 p. 